\title{
Genetic diversity of Streptococcus suis Clinical ISOLATES FROM PIGS AND HUMANS IN ITALY (2003-2007)
}

\author{
M S Princivalli ${ }^{1}$, C Palmieri ${ }^{1}$, G Magi ${ }^{1}$, C Vignaroli ${ }^{1}$, A Manzin², A Camporese ${ }^{3}$, S Barocci ${ }^{4}$, C Magistrali ${ }^{4}$, B Facinelli \\ (b.facinelli@univpm.it) ${ }^{1}$ \\ 1. Department of Biomedical Sciences, Polytechnic University of Marche Medical School, Ancona, Italy \\ 2. Department of Biomedical Sciences and Technologies, Section of Medical Microbiology, \\ University of Cagliari Medical School, Italy \\ 3. Microbiology and Virology Department, S. Maria degli Angeli Regional Hospital, Pordenone, Italy \\ 4. Experimental Zooprophylactic Institute of Umbria and Marche, Perugia, Italy
}

This article was published on 20 August 2009.

Citation style for this article: Gíria M, Rebelo-de-Andrade H, Fernandes T, Pedro S, Freitas G. Report on the measles situation in Portugal. Euro Surveill.

2008;13(42):pii=19010. Available online: http://www.eurosurveillance.org/ViewArticle.aspx?ArticleId=19010

\begin{abstract}
Streptococcus suis, a major porcine pathogen, is emerging as a zoonotic agent capable of causing severe invasive disease in humans exposed to pigs or pork products. S. suis infection is rare in industrialised countries and usually arises as sporadic cases, with meningitis the most common clinical presentation in humans. Recent reports of two cases of meningitis in Sardinia and northeastern Italy prompted this first characterisation of Italian S. suis isolates. Fifty-nine $S$. suis strains, the two recent human strains and 57 swine clinical isolates collected between 2003 and 2007 from different Italian herds and regions, were tested for antimicrobial susceptibility, PCR-screened for virulence and antibiotic resistance genes, and subjected to molecular typing. Phenotypic and genotypic analysis demonstrated an overall high genetic diversity among isolates, the majority of which were resistant to macrolides (78\%) and tetracyclines (90\%). The erm(B), tet( 0$)$, mosaic tet( $/ \mathrm{W} / 32 / 0)$, tet $(\mathrm{W})$, and tet $(\mathrm{M})$ genes were detected. The tet $(0 / \mathrm{W} / 32 / 0)$ gene, the most frequent tet gene after tet( 0$)$, had never been described in the genus Streptococcus before. In addition, a virulent cps2, erm(B) tet(O) clone, belonging to sequence type 1 (ST1) of the ST1 complex, was found to be prevalent and persistent in Italian swine herds. Finally, the two human isolates (both ST1) carrying cps2, $\operatorname{erm}(\mathrm{B})$ and $\operatorname{tet}(\mathrm{W})$ were seen to be closely related to each other.
\end{abstract}

\section{Introduction}

Streptococcus suis, a major porcine pathogen endemic in nearly all countries with a developed swine industry, causes meningitis, pneumonia, arthritis, endocarditis, and septicaemia in pigs [1]. $S$. suis is also emerging as a zoonotic agent capable of causing severe invasive disease in humans exposed to pigs or to pork products $[2,3]$. A carriage state has been documented in pigs, healthy carriers being a source of $S$. suis transmission in herds, mainly through the respiratory route [1]. As discussed in recent reports, the possibility cannot be excluded that humans may also be healthy carriers $[1,3,4]$ and that $S$. suis may become an opportunistic pathogen under particular circumstances such as stress, immunodeficiency or cancer $[1,5]$. Meningitis with possible residual deafness is the most frequent clinical presentation of the infection in humans; septicaemia, pneumonia, endocarditis, arthritis and toxic shock syndrome have also been described. In industrialised countries, $S$. suis disease is rare, albeit probably underdiagnosed, and usually occurs as sporadic cases [2,3]. Most human cases reported so far originated from Southeast Asia, where the disease can be considered endemic and where some outbreaks have occurred [3]. Three major sequence type (ST) clonal complexes (ST1, ST27 and ST87) dominate the population [6]. The virulent ST1 complex, frequently associated with invasive infections, includes sequence type ST1, spread worldwide and recently detected for the first time in Italy [5], and ST7, responsible for several cases of toxic shock syndrome during a recent outbreak in China [7].

The antiphagocytic polysaccharide capsule (encoded by the cps gene) is the major virulence factor of $S$. suis. Thirty-three serotypes based on capsular antigens are currently recognised $[8,9]$. Serotype 2 is responsible for severe infections in swine [1] and is the most common serotype affecting humans worldwide [2]. The small number of human S. suis infections in North America has been linked to the low prevalence of serotype 2 among swine [1]. Serotypes 4, 14 and 16 have also been described in humans [1]. Proposed $S$. suis virulence factors [1], the significance of which is still unknown, include the muramidase released protein MRP (encoded by $m r p$ ), a peptidoglycan-associated protein probably acting as an adhesin and the extracellular protein factor EF (epf), both of which are suitable virulence markers of serotype 2 strains [10] and are also detected in other serotypes [11], a serum opacity factor OFS (ofs), proposed as a virulence trait of cps2 isolates $[12,13]$, suilysin $(s / y)$, a haemolysin with a cytotoxic effect on various cell types [1], and arginine deiminase (arcA), a factor linked to survival in stress conditions [14]. Despite the lack of evidence for a critical role of one or more of these putative virulence factors in virulence, they may nonetheless serve as virulence markers, since MRP, EF, and suilysin are typical of Eurasian strains of the ST1 complex, while they are almost absent in less virulent North American strains [1]. An immune evasion strategy has recently been proposed to account for the allelic variability observed in mrp, epf, and ofs genes $[11,13]$.

A trend toward mounting $S$. suis resistance to macrolides and tetracyclines has been reported worldwide [15-17]. Studies of genetic resistance traits have demonstrated erm(B) (ribosomal methylation) and $\operatorname{mef}(\mathrm{A})$ (active efflux) for macrolide resistance, and tet $(\mathrm{M})$ and tet $(\mathrm{O})$ (both ribosomal protection) for tetracycline 
resistance [18-21]. The tet(W) gene, an emerging determinant commonly found in species inhabiting human and animal intestinal tracts [22], was first detected by our group in a human isolate of S. suis from a case of meningitis in Italy [5].

Overall, three human cases of $S$. suis meningitis have been reported in Italy, one in the 1990s [23] and two quite recently, in the course of little more than a year. The short interval between the last two cases and their arising in distant geographic areas, i.e. north-eastern Italy [24] and Sardinia [5], prompted this first characterisation of Italian S. suis isolates.

\section{Methods}

\section{S. suis strains}

A total of 59 S. suis isolates were studied, two of human and 57 of porcine origin (Table 1). The human isolates, one from Sardinia (SsCA-1: cps2 ST1 erm(B) tet(W)) [5] and the other from north-east Italy [24], here designated as SsUD, were from cerebrospinal fluid (CSF) of two patients with $S$. suis meningitis. All pig isolates were from clinical samples (23 brain, 22 lung and 12 spleen samples) collected in 24 herds in northern and central Italy from 2003 to 2007. They were divided into invasive (brain and spleen isolates: 35 strains) and non-invasive (lung isolates: 22 strains) according to the source of isolation. All strains were isolated on $5 \%$ sheep blood agar (Oxoid Ltd) and identified with ID 32 STREP kit (bioMérieux). Serotyping was performed by slide agglutination using specific antisera (Statens Serum Institute).

\section{Susceptibility testing}

Antimicrobial susceptibility testing by agar disk diffusion and minimal inhibitory concentration (MIC) was carried out according to standard procedures $[25,26]$ (erythromycin and tetracycline antibiotics: Sigma Chemical Co, disks: Oxoid). S. pneumoniae ATCC 49619 was used for quality control. The erythromycin resistance phenotype was determined on the basis of the triple disk test (erythromycin plus clindamycin and josamycin) [27].

\section{Genotyping}

PCR amplification was carried out under published conditions using the oligonucleotide primer pairs and target genes listed in Table 2 [28-33].

Pulsed-Field Gel Electrophoresis (PFGE) was applied to study the genetic diversity of $S$. suis [19,34-36]. Macrorestriction with Smal endonuclease (Roche) and PFGE analysis were performed essentially as described previously [35]. PFGE data were analysed considering each band as a separate putative locus and scoring it as present (1) or absent (0) in each accession. The dendrogram was constructed by use of the Dice coefficient and the unweighted pair group method with arithmetic averages. Genetic relatedness was interpreted according to the criteria of Tenover et al. [37].

A multilocus sequence typing (MLST) scheme for $S$. suis was developed in 2002 [6]. Primers for PCR amplification and sequencing of the housekeeping gene fragments of aroA (EPSP synthase), cpn60 (60-kDa chaperonin), $d p r$ (peroxide resistance), gki (glucose kinase), mutS (DNA mismatch repair enzyme), recA (homologous recombination) and thrA (aspartokinase) were

T A B L L E 1

Streptococcus suis isolates, Italy, 2003-2007 $(\mathbf{n}=59)$

Origin (no. of isolates) Strain (herd*)

\begin{tabular}{|c|c|c|c|}
\hline \multicolumn{4}{|l|}{ Pig (57) } \\
\hline \multirow[t]{6}{*}{ Brain (23) } & V3 (PG/5), v20 (PG/1), V24 (PG/2) & Centre & 2003 \\
\hline & v27 (PG/4), v28 (PG/2), v29 (MC/1), v31 (PG/1), v32 (PG/1), v34 (AR/1), v35 (AR/1), v40 (TR), v42 (PG/1), v36 (PG/1) & Centre & 2004 \\
\hline & v54 (MC/2), v75 (PG/1), v76 (PG/1) & Centre & 2005 \\
\hline & v96 (PG/1), v97 (PG/3) & Centre & 2006 \\
\hline & 170167 (RE), 188509 (RE), 219624 (RE), 202707 (RE) & North & 2007 \\
\hline & $\mathrm{V} 123(\mathrm{PG} / 1)$ & Centre & 2007 \\
\hline \multirow[t]{4}{*}{ Spleen (12) } & V73 (LT) & Centre & 2005 \\
\hline & 45445 (AP/1) & Centre & 2006 \\
\hline & 240370 (RE), 205206 (RE), 210671 (RE), 167757 (RE) & North & 2007 \\
\hline & 20801 (LI), 1303 (AP/1), 22583 (AP/1), 11683 (AP/1), 11707 (PG/7), 13469 (AP/1) & Centre & 2007 \\
\hline \multirow[t]{5}{*}{ Lung (22) } & V21 (PU), v23 (IS), v25 (CH), v26 (PG/2) & Centre & 2003 \\
\hline & 3721 (AP/5), v38 (PG/8) & Centre & 2004 \\
\hline & v92 (PG/3), (AP/1) 27894, (AP/1) 33421, (AP/3) 18237, 30676 (AP/1) & Centre & 2006 \\
\hline & 227794 (RE), 176414 (RE) & North & 2007 \\
\hline & 9649 (PG/6), 22919 (AP/2), 10432 (PG/6), 36774 (AP/1), 30203 (AP/4), 18315 (AN), 1227 (AP/4), 10584 (AP/1), 32457 (AP/1) & Centre & 2007 \\
\hline \multicolumn{4}{|l|}{ Human (2) } \\
\hline \multirow[t]{2}{*}{$\operatorname{CSF}(2)$} & SsUD & North & 2006 \\
\hline & SsCA-1 & Sardinia & 2007 \\
\hline
\end{tabular}

* AN: Ancona, AP: Ascoli Piceno (5 herds), AR: Arezzo, CH: Chieti, IS: Isernia, LI: Livorno, LT: Latina, MC: Macerata (2 herds),

PG: Perugia (8 herds), PU: Pesaro/Urbino, RE: Reggio Emilia, TR: Terni.

CSF: cerebrospinal fluid. 
synthesised according to the primer sequences on the $S$. suis MLST database website (http://ssuis.mlst.net). Sequences were compared with previously observed allelic sequences in the $S$. suis MLST database for identification of ST.

The nucleotide sequences reported here have been submitted to the GenBank/EMBL sequence database and assigned accession numbers FM201280 (ofs (type $^{\text {1s }}$ ), FN357200 (epf ${ }^{915}$ ), FN356743 $(\operatorname{tet}(\mathrm{W}))$ and $\mathrm{FM} 164392(\operatorname{tet}(\mathrm{O} / \mathrm{W} / 32 / 0))$. Sequence similarity searches were carried out using BLAST, available online from the National Center for Biotechnology Information of the National Library of Medicine (http://www.ncbi.nlm.nih.gov).

\section{Results}

\section{Capsular (cps) and virulence-associated genes}

The 59 S. suis isolates were investigated by PCR using primer pairs specific for $c p s 1, c p s 2, c p s 7$, and $c p s 9$, and for virulenceassociated genes $m r p$, epf, ofs, sly, and arcA. Size variants were detected by restriction analysis (epf: HindIII; ofs: $\mathrm{Mbol}$ ) and sequencing (ofs) of PCR products (Table 3). The distributions of $c p s$ and virulence-associated genes are reported in the Figure, and virulence profiles among invasive and non-invasive isolates are shown in Table 4.

T A B L E 2

Streptococcus suis PCR primers and target genes

\begin{tabular}{|c|c|c|c|c|}
\hline Primers & Gene target & Primer sequence $\left(5^{\prime}-3^{\prime}\right)$ & Product length (bp) & Reference \\
\hline \multicolumn{5}{|c|}{ Macrolide resistance genotype } \\
\hline $\begin{array}{l}\text { ERMB } 1 \\
\text { ERMB } 2 \\
\end{array}$ & $\operatorname{erm}(\mathrm{B})$ & $\begin{array}{l}\text { GAAAAGGTACTCAACCAAATA } \\
\text { AGTAACGGTACTTAAATTGTTTAC }\end{array}$ & 639 & {$[28]$} \\
\hline $\begin{array}{l}\operatorname{III}_{10} \\
\operatorname{III}_{8}\end{array}$ & $\operatorname{erm}(\mathrm{TR})$ & $\begin{array}{l}\text { AGGTTATAATGAAACAGA } \\
\text { GCATGACATAAACCTTCA }\end{array}$ & 208 & [29] \\
\hline $\begin{array}{l}\text { MEFA } 1 \\
\text { MEFA } 2\end{array}$ & $m e f(A)$ & $\begin{array}{l}\text { AGTATCATTAATCACTAGTGC } \\
\text { TTCTTCTGGTACTAAAAGTGG }\end{array}$ & 346 & {$[28]$} \\
\hline \multicolumn{5}{|c|}{ Tetracycline resistance genotype } \\
\hline $\begin{array}{l}\text { TETK-up } \\
\text { TETK-rev }\end{array}$ & $\operatorname{tet}(\mathrm{K})$ & $\begin{array}{l}\text { TATTTTGGCTTTGTATTCTTTCAT } \\
\text { GCTATACCTGTTCCCTCTGATAA }\end{array}$ & 1,159 & {$[30]$} \\
\hline $\begin{array}{l}\text { TETL-up } \\
\text { TETL-rev }\end{array}$ & $\operatorname{tet}(\mathrm{L})$ & $\begin{array}{l}\text { ATAAATTGTTTCGGGTCGGTAAT } \\
\text { AACCAGCCAACTAATGACAATGAT }\end{array}$ & 1,077 & {$[30]$} \\
\hline $\begin{array}{l}\text { TETM F } \\
\text { TETM R }\end{array}$ & $\operatorname{tet}(\mathrm{M})$ & $\begin{array}{l}\text { GAACTCGAACAAGAGGAAAGC } \\
\text { ATGGAAGCCCAGAAAGGAT }\end{array}$ & 740 & {$[31]$} \\
\hline $\begin{array}{l}\text { TETO } 1 \\
\text { TETO } 2 \\
\end{array}$ & $\operatorname{tet}(0)$ & $\begin{array}{l}\text { AACTTAGGCATTCTGGCTCAC } \\
\text { TCCCACTGTTCCATATCGTCA }\end{array}$ & 519 & [31] \\
\hline $\begin{array}{l}\text { TETOFF2 } \\
\text { TETOFR3 }\end{array}$ & $\operatorname{tet}(0)$ & $\begin{array}{l}\text { TTGTTTTGGGGCTATTGGAG } \\
\text { TATATGACTTTTCAAGCTG }\end{array}$ & 2,038 & [32] \\
\hline $\begin{array}{l}\text { TETQ F } \\
\text { TETQ R } \\
\end{array}$ & $\operatorname{tet}(Q)$ & $\begin{array}{l}\text { AGAATCTGCTGTTTGCCAGTG } \\
\text { CGGAGTGTCAATGATATTGCA }\end{array}$ & 167 & [33] \\
\hline $\begin{array}{l}\text { TETS F } \\
\text { TETS R }\end{array}$ & $\operatorname{tet}(\mathrm{S})$ & $\begin{array}{l}\text { GAAAGCTTACTATACAGTAGC } \\
\text { AGGAGTATCTACAATATTTAC }\end{array}$ & 168 & [33] \\
\hline $\begin{array}{l}\text { TETT F } \\
\text { TETT R }\end{array}$ & $\operatorname{tet}(\mathrm{T})$ & $\begin{array}{l}\text { AAGGTTTATTATATAAAAGTG } \\
\text { AGGTGTATCTATGATATTTAC }\end{array}$ & 167 & {$[33]$} \\
\hline $\begin{array}{l}\text { TETWF F } \\
\text { TETWF R }\end{array}$ & $\operatorname{tet}(\mathrm{W})$ & $\begin{array}{l}\text { TTGGGGCTGTAAAGGGAGGAC } \\
\text { CTTACATTACCTTCTGA }\end{array}$ & 1948 & {$[32]$} \\
\hline \multicolumn{5}{|c|}{ Virulence-associated factors } \\
\hline $\begin{array}{l}\text { CPS1F } \\
\text { CPS1R }\end{array}$ & $\operatorname{cps} 1 \mathrm{~J}$ & $\begin{array}{l}\text { TGGCTCTGTAGATGATTCTGCT } \\
\text { TGATACGTCAAAATCCTCACCA }\end{array}$ & 637 & {$[11]$} \\
\hline $\begin{array}{l}\text { CPS2F } \\
\text { CPS2R }\end{array}$ & $\operatorname{cps} 2 \mathrm{~J}$ & $\begin{array}{l}\text { TTTGTCGGGAGGGTTACTTG } \\
\text { TTTGGAAGCGATTCATCTCC }\end{array}$ & 498 & {$[11]$} \\
\hline $\begin{array}{l}\text { CPS7F } \\
\text { CPS7R } \\
\end{array}$ & cps7H & $\begin{array}{l}\text { AATGCCCTCGTGGAATACAG } \\
\text { TCCTGACACCAGGACACGTA }\end{array}$ & 379 & {$[11]$} \\
\hline $\begin{array}{l}\text { CPS9F } \\
\text { CPS9R }\end{array}$ & $\mathrm{cps9H}$ & $\begin{array}{l}\text { GGGATGATTGCTCGACAGAT } \\
\text { CCGAAGTATCTGGGCTACTGA }\end{array}$ & 303 & {$[11]$} \\
\hline $\begin{array}{l}\text { MRP1 } \\
\text { MRP2 }\end{array}$ & $m r p$ & $\begin{array}{l}\text { ATTGCTCCACAAGAGGATGG } \\
\text { TGAGCTTTACCTGAAGCGGT }\end{array}$ & $188^{a}$ & {$[11]$} \\
\hline $\begin{array}{l}\text { EPF1 } \\
\text { EPF2 }\end{array}$ & $e p f$ & $\begin{array}{l}\text { CGCAGACAACGAAAGATTGA } \\
\text { AAGAATGTCTTTGGCGATGG }\end{array}$ & $744^{\mathrm{a}}$ & {$[11]$} \\
\hline $\begin{array}{l}\text { OFS-F } \\
\text { OFS-R2 }\end{array}$ & ofs & $\begin{array}{l}\text { GATGTGACTGTCCGCAGAGC } \\
\text { AAAGTACCTGAGCTCCTACA }\end{array}$ & $1,960^{b}$ & {$[13]$} \\
\hline $\begin{array}{l}\text { SLY1 } \\
\text { SLY2 }\end{array}$ & sly & $\begin{array}{l}\text { GCTTGACTTACGAGCCACAA } \\
\text { CCGCGCAATACTGATAAGC }\end{array}$ & 248 & {$[11]$} \\
\hline $\begin{array}{l}\text { ARC-A1 } \\
\text { ARC-A2 }\end{array}$ & $\operatorname{arcA}$ & $\begin{array}{l}\text { TGATATGGTTGCTGCTGGTC } \\
\text { GGACTCGAGGATAGCATTGG }\end{array}$ & 118 & {$[11]$} \\
\hline
\end{tabular}

\footnotetext{
a Reference strain D282; ${ }^{\text {b }}$ Reference strain NIAH11433.
} 
T A B L E 3

The mrp, epf, and ofs gene size variants observed in Streptococcus suis isolates, Italy, 2003-2007

\begin{tabular}{|c|c|c|c|}
\hline Target gene & Size variant & Amplicon size (bp) & References \\
\hline \multicolumn{4}{|l|}{$m r p$} \\
\hline & $m r p$ & 1,148 & {$[11]$} \\
\hline & $m r p^{*}$ & 1,556 & {$[11]$} \\
\hline & $m r p^{s}$ & 747 & [11] \\
\hline \multicolumn{4}{|l|}{$e p f$} \\
\hline & epf & 744 & {$[11]$} \\
\hline & $e p f^{\text {class I }}$ & 3,112 & {$[40]$} \\
\hline & $e p f^{915}$ & 915 & This study \\
\hline \multicolumn{4}{|l|}{ ofs } \\
\hline & ofs $s^{\text {type } 1}$ & 1,960 & [13] \\
\hline & ofstype 1s & 1,636 & This study \\
\hline & ofs type 2 & 2,113 & [13] \\
\hline & ofs type 3a & 1,627 & [13] \\
\hline & ofs type 3b & 1,786 & [13] \\
\hline
\end{tabular}

Three cps genes were detected in 43 of the 59 isolates: $c p s 1$ ( $n=3$ isolates, one invasive), cps2 ( $n=30,23$ invasive, including the two human CSF isolates) and $\operatorname{cps} 9$ ( $\mathrm{n}=10$, eight invasive). In agglutination tests, all cps2 strains showed agglutination with sera specific for serotype 2 . The remaining 16 isolates, of which five were invasive, were negative and are referred to as non-typeable (NT).

The $m r p$ gene (three size variants: $m r p ; m r p^{*}$ and $m r p^{S}$ ) was detected in 47 strains (all 30 cps2 isolates, nine cps9, six NT, and two cps1 isolates); epf (three size variants: epf; epflass I and epf ${ }^{915}$ ) was detected in 31 strains (27 cps2, two cps1 and two

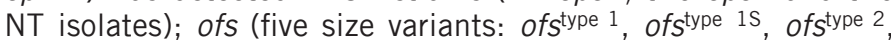
of $s^{\text {type }} 3 \mathrm{a}$ and of stype $^{3 b}$ ) was detected in 40 strains (all 30 cps2, five $c p s 9$, three NT and two cps1 isolates); sly was detected in 52 strains (all cps2 and cps9 isolates, two cps1 and $10 \mathrm{NT}$ isolates), and $\operatorname{arcA}$ was found in all isolates.

\section{Susceptibility testing and detection of resistance genes}

The 59 strains were tested for susceptibility to tetracycline and erythromycin using phenotypic and genotypic methods. Fifty-three strains (90\%) were resistant to tetracycline (MIC 8-64 mg/L) and

\section{F I G U R E}

Similarity index of the 59 Streptococcus suis isolates, Italy, 2003-2007

\begin{tabular}{|c|c|c|c|c|c|c|c|c|c|c|c|c|}
\hline$\%$ SIMI_ARITY & & STRAIN & YEAR & SOURCI & PULSOTYPE & & VIRL & NCE GE & YPE & & RESISTANC & NOTYPE \\
\hline $\begin{array}{c:c}{ }_{50} & 70 \\
\mathbf{S}_{1}\end{array}$ & r & $\mathrm{v} 20(\mathrm{PG} / 1)$ & 2007 & Brain & la & $\operatorname{cps}: 2$ & $m p p$ & $e p f^{t a s} 1$ & $s y=$ & $o f s: \mathrm{w}=1$ & $\operatorname{tet}(0)$ & $\operatorname{em}(\mathrm{B})$ \\
\hline & 量 & & & & & & & & & ( & " & \\
\hline & 资 & VOSG (PGI) & 2003 & $\begin{array}{l}\text { Lung } \\
\text { Lunging }\end{array}$ & $"$ & $"$ & $"$ & $"$ & " & $"$ & $"$ & \\
\hline & I & 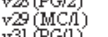 & $\begin{array}{l}2004 \\
2004 \\
2004\end{array}$ & 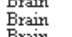 & " & " & " & & " & & & \\
\hline & 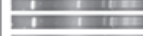 & $\begin{array}{l}\mathrm{V} 1 \mathrm{~V} 1 \mathrm{PG}(\mathrm{PG}) \\
\mathrm{v} 22(\mathrm{PG} / 1)\end{array}$ & 2004 & $\begin{array}{l}\text { Bramu } \\
\text { Brain }\end{array}$ & " & " & $"$ & " & " & " & & \\
\hline & 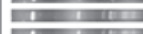 & v34 (ARE) & 2004 & Brain & " & " & " & $"$ & $"$ & $"$ & " & " \\
\hline & 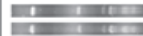 & 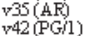 & $\begin{array}{l}2004 \\
2004\end{array}$ & $\begin{array}{l}\text { Brain } \\
\text { Brain }\end{array}$ & " & " & " & " & " & " & & \\
\hline & rin & $\begin{array}{l}v 54(M C D 2) \\
v 73(\mathrm{LT} T)\end{array}$ & $\begin{array}{l}2005 \\
2005\end{array}$ & $\begin{array}{l}\text { Brain } \\
\text { Brpleen } \\
\text { Splats }\end{array}$ & " & $"$ & " & $"$ & " & " & & \\
\hline & 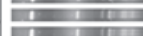 & Y75(PG/1) & $\begin{array}{l}2005 \\
2005 \\
205\end{array}$ & $\begin{array}{l}\text { Brain } \\
\text { Brain } \\
\text { Brain }\end{array}$ & $"$ & " & $"$ & " & " & " & & \\
\hline & 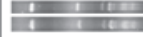 & 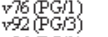 & 2006 & $\begin{array}{l}\text { Brain } \\
\text { Lung }\end{array}$ & " & " & " & " & " & " & & " \\
\hline & 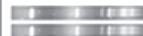 & vas(PG/1) & 2006 & $\begin{array}{l}\text { Brain } \\
\text { Brain }\end{array}$ & " & $"$ & $"$ & " & " & $"$ & & " \\
\hline & 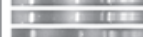 & 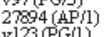 & $\begin{array}{l}2006 \\
2006 \\
2000\end{array}$ & 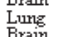 & " & " " & $"$ & " & " & $"$ & " & " \\
\hline & $e^{2}$ & $\begin{array}{l}v_{1230}(\mathrm{PG} / \mathrm{GI}) \\
20001(\mathrm{LI})\end{array}$ & 2007 & $\begin{array}{l}\text { Bram } \\
\text { Spleen }\end{array}$ & " & " & " & " & " & " & & \\
\hline & tinn & $\begin{array}{l}227794(\mathrm{R}) \\
24030(\mathrm{RE})\end{array}$ & 2000 & $\begin{array}{l}\text { Lumg } \\
\text { Spleen }\end{array}$ & " & " & $"$ & & " & & & \\
\hline & 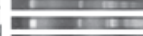 & $\begin{array}{l}200206(\mathrm{R} R) \\
33421(\mathrm{AP} / 1)\end{array}$ & 2006 & $\begin{array}{l}\text { Spleen } \\
\text { Lung }\end{array}$ & 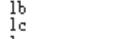 & 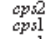 & 吕 $m_{m} p_{s}^{s}$ & 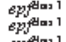 & sy & 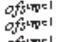 & $\begin{array}{l}\operatorname{tet}(0) \\
\operatorname{tent}(W)\end{array}$ & $\operatorname{erm}(\mathrm{B})$ \\
\hline & 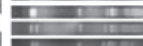 & 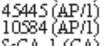 & $\begin{array}{l}2006 \\
2007 \\
2007\end{array}$ & 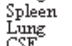 & $\begin{array}{l}\text { lc } \\
\text { id } \\
\text { id }\end{array}$ & $\begin{array}{l}c p s 1 \\
c p 52 \\
c p 25\end{array}$ & $\begin{array}{c}m p^{3} \\
m p \\
m p m\end{array}$ & 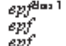 & $\begin{array}{c}\text { siy } \\
s y \\
y y\end{array}$ & 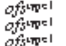 & tetwi & $\operatorname{exm}\left(\mathrm{B}_{\mathrm{B}}\right)$ \\
\hline & 달 & 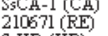 & 2007 & Spleen & le & $\begin{array}{l}c p s 2 \\
c p s q \\
c o p\end{array}$ & $\begin{array}{l}m p \\
m p \\
m p\end{array}$ & ept & $\begin{array}{l}3, y \\
s y \\
s y\end{array}$ & 0.05 wet & $\begin{array}{l}\operatorname{tat}(1) \\
\operatorname{tet}(0)\end{array}$ & $\operatorname{em}(\mathrm{B}) \mathrm{s}$ \\
\hline & ( & & 2000 & $\begin{array}{l}\text { CSF } \\
\text { Brain }\end{array}$ & & cpsi & 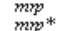 & epf & 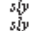 & offirmels & tet(1) & $\operatorname{em}(B)$ \\
\hline & 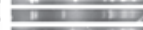 & & 2000 & Brain & ih & cps 2 & $m p$ & epf & siy & ofs $\mathrm{w} \mathrm{e}=1$ & tetio) & em(B) \\
\hline & 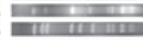 & 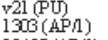 & 2007 & $\begin{array}{l}\text { Lung } \\
\text { Spleen }\end{array}$ & $\begin{array}{l}2 \mathrm{a} \\
2 \mathrm{~b}\end{array}$ & $\begin{array}{l}c p s, \\
c p, s \\
c p s\end{array}$ & $\stackrel{m}{m p} *$ & & 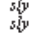 & & 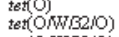 & $\begin{array}{l}e m(B) \\
e m(B)\end{array}$ \\
\hline & 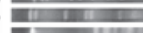 & $\begin{array}{l}32457(\mathrm{AP} / 1) \\
10101\end{array}$ & 2007 & Lung & 3 & NT & - & & $3 y$ & & $\operatorname{tet}(0 / \mathrm{W} / 32 / 0)$ & $\operatorname{em}(\mathrm{B})$ \\
\hline & $\frac{1}{10}$ & 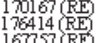 & $\begin{array}{l}2007 \\
2007 \\
2007\end{array}$ & $\begin{array}{l}\text { Brain } \\
\text { Lumg } \\
\text { Sulcon }\end{array}$ & $\begin{array}{l}4 \\
5 \\
5 \\
\end{array}$ & 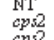 & $m p$ & $:$ & $\begin{array}{l}s, y \\
s y \\
\text { s. }\end{array}$ & 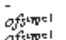 & 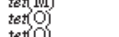 & \\
\hline & II: & 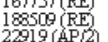 & $\begin{array}{l}2007 \\
2000\end{array}$ & $\begin{array}{l}\text { Spetin } \\
\text { Brain } \\
\text { Lumg }\end{array}$ & 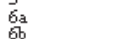 & 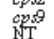 & $m p *$ & : & $3 y$ & of & 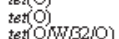 & unlnown \\
\hline & E & & 2006 & Lung & $7 \mathrm{a}$ & $\mathrm{NT}$ & & & siy & & & em(B) \\
\hline & (1) & $30003(\mathrm{AP} / 4)$ & $\begin{array}{l}2000 \\
2003\end{array}$ & $\operatorname{Lum} 8$ & $7 \mathrm{~b}$ & $\mathrm{NT}$, & $m p *$ & & & & $\operatorname{tet}(0 / \mathrm{W} / 2 \mathrm{O})$ & eming \\
\hline & m & 36774 (AP/1) & 2007 & Lung & $8 \mathrm{~b}$ & NT & - & & & & & $\operatorname{em}(B)$ \\
\hline & Fiming & $\begin{array}{l}1227 \mathrm{AP} 4 \mathrm{P}) \\
10432 \text { (PGG) }\end{array}$ & 2007 & $\begin{array}{l}\text { Lung } \\
\text { Lung }\end{array}$ & $\begin{array}{l}9 \mathrm{~g} \\
9 \mathrm{~g}\end{array}$ & 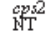 & $m p$ & eppops & $\begin{array}{ll}3 y y \\
3 y y\end{array}$ & 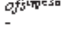 & $\begin{array}{l}\operatorname{teter}(1) \\
\operatorname{tet}(0)\end{array}$ & $\operatorname{em}(\mathrm{B})$ \\
\hline & 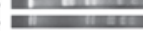 & $\begin{array}{l}219624(\mathrm{RE}) \\
11683(\mathrm{AP} / 1)\end{array}$ & 2007 & $\begin{array}{l}\text { Brain } \\
\text { Spleen }\end{array}$ & $\begin{array}{l}10 a \\
10 b\end{array}$ & $\begin{array}{l}\mathrm{NT} \\
c p s 9\end{array}$ & $\stackrel{m p}{m p} *$ & - & $\begin{array}{l}s, y \\
s y \\
s y\end{array}$ & offor & $\operatorname{tet}(0, N 3210)$ & $\operatorname{com}(B)$ \\
\hline & 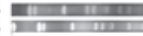 & $\begin{array}{l}3721(\mathrm{APS}) \\
\mathrm{Y} 25(\mathrm{CH})\end{array}$ & ${ }_{2004}^{2004}$ & $\begin{array}{l}\text { Lung } \\
\text { Lung }\end{array}$ & $\begin{array}{l}10 \mathrm{c} \\
100 \mathrm{~d}\end{array}$ & NT & $\begin{array}{c}m p \\
m p \\
m p\end{array}$ & & 滛 & 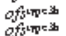 & 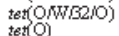 & emi(B) \\
\hline & min & & 2004 & Lung & $\begin{array}{ll}100 \\
100\end{array}$ & cops & $\underset{m p}{m p}$ & : & $3 y$ & 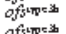 & & \\
\hline & 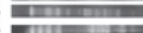 & (AN) & 2007 & Lung & & $\begin{array}{l}\text { NT } \\
\text { ins }\end{array}$ & in* & & $33 y$ & ofturese? & 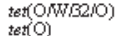 & $\begin{array}{l}\operatorname{exm}(B) \\
\operatorname{em}(\mathrm{B})\end{array}$ \\
\hline & III & & 2007 & Lung & $12 \mathrm{~b}$ & NT & - & ing & & fintes & & \\
\hline & (11) & 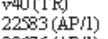 & 2007 & Spleen & $14 a$ & $\begin{array}{l}141 \\
c p s 9\end{array}$ & $\operatorname{mat}$ & & $s y$ & & 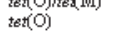 & $\operatorname{erm}(\mathrm{B})$ \\
\hline-5 & Fin & $\begin{array}{l}3076,(\mathrm{AP} / 1) \\
11707 \mathrm{PGG})\end{array}$ & $\begin{array}{l}2006 \\
2007 \\
2007\end{array}$ & $\begin{array}{l}\text { Lumg } \\
\text { Spleen } \\
\text { SIlone }\end{array}$ & 列 & 䇥T & & & syy & & $\operatorname{tet}(0)$ & unlnown \\
\hline
\end{tabular}

For each isolate, the year and the source of isolation and the virulence and resistance genotypes are shown. Pulsed-field gel electrophoresis pulsotypes sharing $>70 \%$ similarity were grouped into clusters (gray).

Unknown: neither erm (A) nor erm(B) nor mef(A).

SCCA-1 and SSUD are the two human isolates 
46 (78\%) were constitutively resistant to erythromycin (MIC >128 $\mathrm{mg} / \mathrm{L}: \mathrm{n}=44$, including SsCA-1; MIC 4 mg/L: $\mathrm{n}=2$, including SsUD). All erythromycin-resistant strains were also tetracycline-resistant. The erm(B) gene was the only erythromycin resistance determinant (Figure), found in 44 of 46 erythromycin-resistant strains. Neither $\operatorname{erm}(A)$ nor $\operatorname{mef}(A)$ were detected in the two erythromycin-resistant ( $\mathrm{MIC}>128 \mathrm{mg} / \mathrm{L}$ ) erm(B)-negative strains. Tetracycline resistance genes were distributed as follows: tet $(0)(n=38)$, tet $(0 / W / 32 / 0)$ $(n=8), \operatorname{tet}(W)(n=5) ; \operatorname{tet}(M)(n=1)$, and $\operatorname{tet}(0) / \operatorname{tet}(M)(n=1)$.

The presence of the mosaic gene was suspected from incongruent findings in PCR experiments, where a 519 bp amplicon was obtained in 38 strains using primers internal to tet(0) (TETO1 and TETO2), and a 2,038 bp amplicon was obtained in 46 strains (of which eight were negative when internal primers were used) using full-length tet( 0$)$ primers (TETOFF2 and TETOFR3). In the latter strains the presence of the mosaic gene tet(0/W/32/0) was confirmed by Alul and Hinfl restriction analysis and sequencing of PCR products. Sequence analysis (FM164392) revealed that this gene was $99 \%$ identical to the tetracycline resistance gene tet(O/W/32/O) (EF065523.1) of an uncultured bacterium isolated from pig faeces [32]. The tet(W) gene was detected in three pig isolates and in both human isolates by Hinfl restriction analysis of the amplicons obtained with the tetWFF and tetWFR primer pair and sequencing. Sequence analysis (FN356743) disclosed that

\section{T A B L E 4}

Virulence-associated gene profiles in Streptococcus suis isolates, Italy, 2003-2007 $(n=59)$

\begin{tabular}{|c|c|c|}
\hline Profile & Invasive & Non-invasive \\
\hline cps2 isolates $(n=30)$ & 23 & 7 \\
\hline$m r p$ epf class I ofs type 1 sly arcA & 19 & 4 \\
\hline mrp epf ofs type 1 sly arc $A$ & $2^{*}$ & 1 \\
\hline mrp epf ofs type 1 s sly arcA & 1 & - \\
\hline$m r p$ ofs type ${ }^{1}$ sly arc $A$ & 1 & 1 \\
\hline$m r p$ ofs type 3 a $\operatorname{arc} A$ & - & 1 \\
\hline cps1 isolates $(n=3)$ & 1 & 2 \\
\hline$m r p^{s}$ epfclass I ofs type I sly arcA & 1 & 1 \\
\hline $\operatorname{arc} A$ & - & 1 \\
\hline cps9 isolates $(n=10)$ & 8 & 2 \\
\hline$m r p^{*}$ ofs type 2 sly $\operatorname{arcA}$ & 3 & - \\
\hline mrp ofs type 3b sly arcA & 1 & 1 \\
\hline mrp sly $\operatorname{arc} A$ & 1 & 1 \\
\hline$m r p^{\star}$ sly $\operatorname{arc} A$ & 2 & - \\
\hline $\operatorname{sly} \operatorname{arc} A$ & 1 & - \\
\hline${ }^{\text {aNT }}$ isolates $(n=16)$ & 5 & 11 \\
\hline$m r p$ epf ${ }^{915}$ ofs type 2 sly arcA & 1 & - \\
\hline mrp ofs type 3b sly arcA & - & 2 \\
\hline epf ${ }^{915}$ sly $\operatorname{arc} A$ & - & 1 \\
\hline mrp sly arcA & 1 & - \\
\hline$m r p^{\star} \operatorname{arc} A$ & - & 2 \\
\hline sly $\operatorname{arc} A$ & 3 & 2 \\
\hline $\operatorname{arc} A$ & - & 4 \\
\hline
\end{tabular}

a NT: non-typeable (neither cps1, nor 2, 7 or 9). * Human isolates it was $99 \%$ identical to the tetracycline resistance gene tet(W) (DQ519395.1) of a porcine isolate of Arcanobacterium pyogenes [38].

\section{PFGE typing and MLST}

All strains were PFGE-typed after Smal digestion of total DNA. Thirty-four different pulsotypes were detected and grouped into 14 PFGE types (types 1 to 14 ) on the basis of a cut-off of $70 \%$ similarity (Figure). PFGE type 1 accounted for $52 \%$ of isolates and comprised eight pulsotypes (types a to h), of which pulsotype $1 \mathrm{a}$ was shared by 22 pig isolates collected from 10 different herds in northern and central Italy in the period from 2003 to 2007. Pulsotype 1d was shared by the human strain SsCA-1 (isolated in 2007) and the pig isolate 10584 (isolated in 2006), and pulsotype $1 \mathrm{f}$ was displayed by the human strain SsUD. Comparison of $1 \mathrm{~d}$ with both pulsotypes $1 \mathrm{a}$ and $1 \mathrm{f}$ yielded a two-band difference, and comparison of $1 \mathrm{a}$ with $1 \mathrm{f}$ a three-band difference. MLST of strains v20 (chosen as representative of pulsotype 1a), SsCA-1 (1d), and SsUD (1f) identified the same allelic profile, corresponding to ST1.

\section{Clones}

The distribution of $c p s$ genes, virulence-associated genes, and tetracycline and erythromycin resistance determinants among the 59 S. suis strains subdivided by PFGE types and pulsotypes is detailed in the Figure. S. suis isolates with a unique combination of a given PFGE pulsotype, a given cps gene, a given virulence profile, and a given resistance genotype and phenotype were considered to represent a clone. According to this criterion, 34 different clones, corresponding to the 34 different pulsotypes, were recognised, 32 of which were found among the 57 pig isolates (Figure). A major cps2 swine clone (clone 1a: mrp, epfclass I; ofstype 1, sly, arcA; tet $(0) \operatorname{erm}(B))$ accounted for $37 \%$ of the 59 isolates. Moreover, clones 1d (mrp, epf; ofs type 1, sly, $\operatorname{arcA}$; tet $(\mathrm{W}) \operatorname{erm}(\mathrm{B}))$ and $1 \mathrm{f}$ (mrp, epf; ofs type $1 \mathrm{~s}^{\mathrm{s}}$ sly, $\operatorname{arcA}$; tet(W) erm(B)), containing the two human isolates (SsCA-1 and SsUD, respectively), were seen to be closely related.

\section{Discussion and conclusion}

This is the first study of virulence and resistance traits in swine and human strains of $S$. suis in Italy. The cps genes coding for the capsular polysaccharide as well as mrp, epf, ofs, and sly genes were investigated. The most prevalent capsular gene was cps2, followed by cps9 and cps1. The cps 2 and $c p s 9$ genes were detected more frequently among invasive isolates; NT isolates were more frequent among non-invasive isolates.

In the present study, virulence-associated genes mrp, epf, sly, and ofs were found in a large proportion of isolates, including NT isolates. The arcA gene was seen in all strains, confirming previous studies [1]. The epf gene was not detected in cps9 strains, in line with a previous report [11], whereas the recently described ofs gene $[12,13]$ was detected not only in all cps2 but also in some cps1, $c p s 9$, and NT strains. Human and pig cps2 isolates carrying mrp and epf, were detected. Interestingly, strains carrying mrp and epf have been previously proved to induce meningitis and septicaemia in experimentally infected pigs [39]. Moreover, cps2 strains carrying mrp epflass I and ofstype 1 were detected in pig isolates. The size variants mrp and epflass I have been described in human isolates in Europe [40] and recently found in invasive cps2 swine clones from Europe and Brazil $[11,41]$. The size variant ofs $s^{\text {type }} 1$ has been found to be associated with the ST1 complex [13]. Other profiles, such as cps1 mrpS- and cps9 $\mathrm{mrp}^{*}$ - have also been described in isolates from diseased pigs in European countries $[10,11]$. 
The finding that invasive and non-invasive isolates share identical virulence profiles seems to support the hypothesis that other, as yet unknown virulence factors are involved in $S$. suis pathogenesis $[1,3]$. The high allele variability of these genes was confirmed by detection of several size variants of mrp, epf, and ofs, of which some had previously been described $[10,11,13,40]$ and some were new (epf $f^{15}$ and of $s^{\text {type } 1 S}$ ).

High rates of resistance to macrolides and tetracyclines suggested widespread resistance to these antibiotics in Italy. In Europe, rising rates of resistance have been attributed to intensive use by swine breeders of the macrolide-class antibiotic tylosin as a growth promoter and of tetracycline as a therapeutic agent [15]. Co-resistance to macrolides and tetracyclines can be explained by the fact that tetracycline and erythromycin resistance determinants are often linked on mobile genetic elements [42].

All strains were PCR screened for $\operatorname{erm}(A), \operatorname{erm}(B)$, and mef(A). Neither $\operatorname{erm}(A)$ nor mef(A) were detected. The erm(B) gene was found in all but two erythromycin-resistant pig strains, confirming its prevalence in $S$. suis in Europe [18,19]. A possible explanation for the erythromycin-resistant, $\operatorname{erm}(\mathrm{A})$-, $\operatorname{erm}(\mathrm{B})$ - and $\operatorname{mef}(\mathrm{A})$-negative strains could be an erythromycin resistance determinant previously unreported in S. suis [21]. The presence of erm(B) in both human isolates is consistent with its dissemination in the Italian swine population. The genetic basis of erythromycin resistance in human $S$. suis isolates has barely been investigated $[5,21]$. The very recent paper by Chu et al. [21] describes the prevalence of mef(A) in isolates from Hong Kong. Interestingly, all mef(A) isolates belonged to ST7 (endemic in Asia) whereas the only erm(B) strain belonged to ST1 (spread worldwide, including in Europe) [21].

The tet $(\mathrm{M})$ and tet $(\mathrm{O})$ genes are common resistance determinants in S. suis, found worldwide both in pig and in human isolates $[19,20]$. In this study, four tet genes, all coding for ribosomal protection proteins (http://faculty.washington.edu/marilynr/), were found in the Italian $S$. suis population. While tet( 0$)$ was prevalent, tet( $(\mathrm{M})$ was, inexplicably, almost absent. In addition tet( $\mathrm{W})$, and the mosaic tet( $0 / \mathrm{W} / 32 / 0)$, the tet gene found most frequently in pig isolates after tet( $(0)$, were detected. The tet( $\mathrm{W})$ gene is associated with tetracycline resistance in a wide range of bacterial species, including obligate anaerobic rumen bacteria and isolates from human gut and oral mucosa. tet(W) was first detected in $S$. suis by our group in the human isolate SsCA-1 [5], and then here in the other human strain (SSUD) and in some pig isolates. These data suggest that tet( $\mathrm{W})$ could be widespread in S. suis.

The mosaic gene tet( $0 / \mathrm{W} / 32 / 0)$ has not been described in the genus Streptococcus before. Mosaic tet genes, originating from tet $(0)$ and tet(W), were first detected in 2003 in anaerobic Gramnegative Megasphaera elsdenii from swine intestine $[43,44]$. Other mosaic genes, also comprising tet(32), were later detected in Clostridium difficile [45]. Initially thought to be confined to a small group of anaerobic bacteria [22], mosaic tet genes have now been found to be abundant in human and animal faecal samples [32] and have also been detected in Bifidobacterium thermophilum and Lactobacillus johnsonii isolates [46]. Further studies on the genetic elements carrying tet genes are warranted to explain the atypical tet distribution observed in Italian S. suis isolates.

Overall, the $S$. suis pig isolates demonstrated a high genetic diversity that correlates with a wide distribution of $S$. suis in Italy. In a heterogeneous background population, an identical virulence and resistance profile (cps2 mrp epf class I of $s^{\text {type } 1}$ sly erm(B) tet(0)) and pulsotype were shared by more than a third of swine isolates, collected between 2003 and 2007 from different Italian herds and regions, demonstrating the presence and persistence of a dominant clone, $1 \mathrm{a}$.

The results further revealed that the two human isolates shared a number of common or related features, i.e. both were serotype 2 and harboured $c p s 2$, both were resistant to erythromycin (MIC $4 \mu \mathrm{g} / \mathrm{ml}$ and $>128 \mu \mathrm{g} / \mathrm{ml}$, respectively) and contained the erm(B) gene, and both were resistant to tetracycline (MIC $16 \mu \mathrm{g} / \mathrm{ml}$ ) and contained the tet(W) gene. Moreover, while sharing the same $m r p$ and epf variants as well as sly, the two human isolates SsUD and SsCA-1 bore two different ofs variants, respectively ofs $s^{\text {type }} 1$ and of $s^{\text {type } 1 S}$, a new variant with a 324 bp deletion in the ofs $s^{\text {type }}{ }^{1}$ coding sequence.

According to Tenover's criteria [37], a close relatedness between SsUD and SSCA-1 and between each human isolates and the dominant swine clone was documented by PFGE analysis which yielded pulsotypes with a difference in only two or three bands. MLST analysis assigned clones $1 \mathrm{a}$ and $1 \mathrm{f}$ (SsUD) to ST1 of the highly virulent ST1 complex, as previously demonstrated also for SsCA-1 (clone 1d) [5]. Overall, our data show that typical Eurasian strains, i.e. strains carrying genes coding for MRP, EF, and suilysin and belonging to the ST1 complex [1], are widespread in Italy.

In conclusion, this study demonstrated a high genetic diversity of Italian S. suis isolates, with a prevalent $c p s 2$, erm(B), tet(O) ST1 clone persistent in the swine population. It also demonstrated a close relatedness between two recently isolated cps2 erm(B) and tet(W) ST1 human strains and between human isolates and the dominant swine clone. Finally, it is the first report to demonstrate tet( $(0 / \mathrm{W} / 32 / 0)$ in $S$. suis and suggests that mosaic tet genes should be sought in $S$. suis and in other streptococci.

\section{Acknowledgements}

This work was partly supported by a grant from the Italian Ministry of Education, University and Research.

\section{References}

1. Gottschalk M, Segura M, Xu J. Streptococcus suis infections in humans: the Chinese experience and the situation in North America. Anim Health Res Rev. 2007;8(1):29-45.

2. Lun ZR, Wang QP, Chen XG, Li AX, Zhu XQ. Streptococcus suis: an emerging zoonotic pathogen. Lancet Infect Dis. 2007;7(3):201-9.

3. Wertheim HF, Nghia HD, Taylor W, Schultsz C. Streptococcus suis: an emerging human pathogen. Clin Infect Dis. 2009;48(5):617-25.

4. Smith TC, Capuano AW, Boese B, Myers KP, Gray GC. Exposure to Streptococcus suis among US swine workers. Emerg Infect Dis. 2008;14(12):1925-7.

5. Manzin A, Palmieri C, Serra C, Saddi B, Princivalli MS, Loi G et al. Streptococcus suis meningitis without evidence of animal contact, Italy. Emerg Infect Dis. 2008;14(12):1946-8.

6. King SJ, Leigh JA, Heath PJ, Luque I, Tarradas C, Dowson CG et al. Development of a multilocus sequence typing scheme for the pig pathogen Streptococcus suis: identification of virulent clones and potential capsular serotype exchange. J Clin Microbiol. 2002;40(10):3671-80.

7. Ye C, Zhu X, Jing H, Du H, Segura M, Zheng H et al. Streptococcus suis sequence type 7 outbreak, Sichuan, China. Emerg Infect Dis. 2006;12(8):1203-8.

8. Staats JJ, Feder I, Okwumabua 0, Chengappa MM. Streptococcus suis: past and present. Vet Res Commun. 1997;21(6):381-407.

9. Hill JE, Gottschalk M, Brousseau R, Harel J, Hemmingsen SM, Goh SH. Biochemical analysis, cpn60 and 16S rDNA sequence data indicate that Streptococcus suis serotypes 32 and 34 , isolated from pigs, are Streptococcus orisratti. Vet Microbiol. 2005;107(1-2):63-9. 
10. Wisselink HJ, Smith HE, Stockhofe-Zurwieden N, Peperkamp K, Vecht U. Distribution of capsular types and production of muramidase-released protein (MRP) and extracellular factor (EF) of Streptococcus suis strains isolated from diseased pigs in seven European countries. Vet Microbiol. 2000;74(3):237-48.

11. Silva LM, Baums CG, Rehm T, Wisselink HJ, Goethe R, Valentin-Weigand P. Virulence-associated gene profiling of Streptococcus suis isolates by PCR. Vet Microbiol. 2006;115(1-3):117-27.

12. Baums CG, Kaim U, Fulde M, Ramachandran G, Goethe R, Valentin-Weigand P. Identification of a novel virulence determinant with serum opacification activity in Streptococcus suis. Infect Immun. 2006;74(11):6154-62.

13. Takamatsu D, Osaki M, Tharavichitkul P, Takai S, Sekizaki T. Allelic variation and prevalence of serum opacity factor among the Streptococcus suis population. J Med Microbiol. 2008;57(Pt4):488-94.

14. Gruening P, Fulde M, Valentin-Weigand P, Goethe R. Structure, regulation, and putative function of the arginine deiminase system of Streptococcus suis. J Bacteriol. 2006;188(2):361-9

15. Wisselink HJ, Veldman KT, Van den Eede C, Salmon SA, Mevius DJ. Quantitative susceptibility of Streptococcus suis strains isolated from diseased pigs in seven European countries to antimicrobial agents licensed in veterinary medicine. Vet Microbiol. 2006;113(1-2):73-82

16. Hendriksen RS, Mevius DJ, Schroeter A, Teale C, Jouy E, Butaye $P$ et al. Occurrence of antimicrobial resistance among bacterial pathogens and indicator bacteria in pigs in different European countries from year 2002 2004: the ARBAO-II study. Acta Vet Scand. 2008;13(1);50:19.

17. Zhang C, Ning Y, Zhang Z, Song L, Qiu H, Gao H. In vitro antimicrobial susceptibility of Streptococcus suis strains isolated from clinically healthy sows in China. Vet Microbiol. 2008;131(3-4):386-92.

18. Martel A, Baele M, Devriese LA, Goossens H, Wisselink HJ, Decostere A, et al. Prevalence and mechanism of resistance against macrolides and lincosamides in Streptococcus suis isolates. Vet Microbiol. 2001;83(3):287-97.

19. Tian Y, Aarestrup FM, Lu CP. Characterization of Streptococcus suis serotype 7 isolates from diseased pigs in Denmark. Vet Microbiol. 2004;103(1-2):55-62.

20. Ye C, Bai X, Zhang J, Jing H, Zheng H, Du H, et al. Spread of Streptococcus suis sequence type 7, China. Emerg Infect Dis 2008;14(5):787-91

21. Chu YW, Cheung TK, Chu MY, Tsang VY, Fung JT, Kam KM, et al. Resistance to tetracycline, erythromycin and clindamycin in Streptococcus suis serotype 2 in Hong Kong. Int J Antimicrob Agents. 2009;34(2):181-2.

22. Roberts MC. Update on acquired tetracycline resistance genes. FEMS Microbiol Lett. 2005;245(2):195-203.

23. Perseghin P, Bezzi G, Troupioti P, Gallina M. Streptococcus suis meningitis in an Italian blood donor. Lancet. 1995;346(8985):1305-6.

24. Camporese A, Tizianel G, Bruschetta G, Cruciatti B, Pomes A. Human meningitis caused by Streptococcus suis: the first case report from north-eastern Italy. Infez Med. 2007;15(2):111-4 [Article in Italian].

25. Clsi.org [homepage on the internet]. Wayne, PA. Clinical and Laboratory Standards Institute. Performance standards for antimicrobial susceptibility testing. 18th informational supplement. Matthew A Wikler et al. Clinical and Laboratory Standards Institute; 2008. CLSI Document M100-S18.

26. Clsi.org [homepage on the internet]. Wayne, PA. Clinical and Laboratory Standards Institute. Performance standards for antimicrobial disk and dilution susceptibility tests for bacteria isolated from animals, approved standard, 2nd Edition. Clinical and Laboratory Standards Institute; 2002. CLSI Document M31-A2. Available from: http://www.clsi.org/source/orders/free/ m31-a3.pdf

27. Giovanetti E, Montanari MP, Mingoia M, Varaldo PE. Phenotypes and genotypes of erythromycin-resistant Streptococcus pyogenes strains in Italy and heterogeneity of inducibly resistant strains. Antimicrob Agents Chemother. 1999;43(8):1935-40.

28. Sutcliffe J, Tait-Kamradt A, Wondrack L. Streptococcus pneumoniae and Streptococcus pyogenes resistant to macrolides but sensitive to clindamycin: a common resistance pattern mediated by an efflux system. Antimicrob Agents Chemother. 1996;40(8):1817-24.

29. Seppälä H, Skurnik M, Soini H, Roberts MC, Huovinen P. A novel erythromycin resistance methylase gene (ermTR) in Streptococcus pyogenes. Antimicrob Agents Chemother. 1998;42(2):257-62.

30. Trzcinski K, Cooper BS, Hryniewicz W, Dowson CG. Expression of resistance to tetracyclines in strains of methicillin-resistant Staphylococcus aureus. J Antimicrob Chemother. 2000;45(6):763-70.

31. Olsvik B, Olsen I, Tenover FC. Detection of tet(M) and tet(0) using the polymerase chain reaction in bacteria isolated from patients with periodontal disease. Oral Microbiol Immunol. 1995;10(4):87-92.

32. Patterson AJ, Rincon MT, Flint HJ, Scott KP. Mosaic tetracycline resistance genes are widespread in human and animal fecal samples. Antimicrob Agents Chemother. 2007;51(3):1115-8.
33. Aminov RI, Garrigues-Jeanjean N, Mackie RI. Molecular ecology of tetracycline resistance: development and validation of primers for detection of tetracycline resistance genes encoding ribosomal protection proteins. Appl Environ Microbiol. 2001;67(1):22-32.

34. Vela AI, Goyache J, Tarradas C, Luque I, Mateos A, Moreno MA et al. Analysis of genetic diversity of Streptococcus suis clinical isolates from pigs in Spain by pulsed-field gel electrophoresis. J Clin Microbiol. 2003;41(6):2498-502.

35. Berthelot-Hérault F, Marois C, Gottschalk M, Kobisch M. Genetic diversity of Streptococcus suis strains isolated from pigs and humans as revealed by pulsed-field gel electrophoresis. J Clin Microbiol. 2002;40(2):615-9.

36. Allgaier A, Goethe R, Wisselink HJ, Smith HE, Valentin-Weigand P. Relatedness of Streptococcus suis isolates of various serotypes and clinical backgrounds as evaluated by macrorestriction analysis and expression of potential virulence traits. J Clin Microbiol. 2001;39(2):445-53.

37. Tenover FC, Arbeit RD, Goering RV, Mickelsen PA, Murray BE, Persing DH et al. Interpreting chromosomal DNA restriction patterns. J Clin Microbiol. 1995;33(9):2233-9.

38. Billington SJ, Jost BH. Multiple genetic elements carry the tetracycline resistance gene tet(W) in the animal pathogen Arcanobacterium pyogenes. Antimicrob Agents Chemother. 2006;50(11):3580-7

39. Smith HE, Vecht U, Wisselink HJ, Stockhofe-Zurwieden N, Biermann Y, Smits MA. Mutants of Streptococcus suis types 1 and 2 impaired in expression of muramidase-released protein and extracellular protein induce disease in newborn germfree pigs. Infect Immun. 1996;64(10):4409-12.

40. Smith HE, Reek FH, Vecht U, Gielkens AL, Smits MA. Repeats in an extracellular protein of weakly pathogenic strains of Streptococcus suis type 2 are absent in pathogenic strains. Infect Immun. 1993;61(8):3318-26.

41. Martinez G, Pestana de Castro AF, Ribeiro Pagnani KJ, Nakazato G, Dias da Silveira W, Gottschalk M. Clonal distribution of an atypical MRP+, EF*, and suilysin+ phenotype of virulent Streptococcus suis serotype 2 strains in Brazil. Can J Vet Res. 2003;67(1):52-5

42. Roberts MC. Update on macrolide-lincosamide-streptogramin, ketolide, and oxazolidinone resistance genes. FEMS Microbiol Lett. 2008;282(2):147-59.

43. Stanton TB, Humphrey SB. Isolation of tetracycline-resistant Megasphaera elsdenii strains with novel mosaic gene combinations of tet( 0 ) and tet(W) from swine. Appl Environ Microbiol. 2003;69(7):3874-82.

44. Stanton TB, McDowall JS, Rasmussen MA. Diverse tetracycline-resistant genotypes of Megasphaera elsdenii strains selectively cultured from swine feces. Appl Environ Microbiol. 2004;70(6):3754-7.

45. Spigaglia P, Barbanti F, Mastrantonio P. Tetracycline resistance gene tet(W) in the pathogenic bacterium Clostridium difficile. Antimicrob Agents Chemother. 2008;52(2):770-3.

46. van Hoek AH, Mayrhofer S, Domig KJ, Flórez AB, Ammor MS, Mayo B et al, Mosaic tetracycline resistance genes and their flanking regions in Bifidobacterium thermophilum and Lactobacillus johnsonii. Antimicrob Agents Chemother. 2008;52(1):248-52. 